\title{
Asymmetric Variable Universe Adaptive Landing Fuzzy Controller for Carrier-Based Aircraft
}

\author{
Hui Li, Xiao-dong Su and Hai-tao Jiang \\ School of Computer and Information Engineering \\ Harbin University of Commerce \\ hrbcu_lh@163.com
}

\begin{abstract}
During the process of aircraft landing, the control manners of carrier-based aircraft are always used to study. This is one of the important ways to avoid pilot-aircraft adverse coupling. In order to keep the accuracy of landing, this paper presents an improved asymmetric variable universe adaptive landing fuzzy controller with Safe Flight Area during approach for carrier-based aircraft. By means of universe-conversion factors and contraction - expansion factors, universe of discourse can be modified online, and fuzzy rules can reproduce automatically to adapt to the modified universe of discourse. The model simulation results indicate the better performance of the new method in comparison with the traditional controller with more accuracy and practicability.
\end{abstract}

Keywords: Asymmetric Variable Universe Adaptive Fuzzy Control; UniverseConversion Factor; Safe Flight Area; Carrier-Based Aircraft

\section{Introduction}

To ensure landing on angled-deck of carrier safely and quickly during manual landing process, the pilot of carrier-based aircraft should be control flight path and attitude precisely. Landing on an aircraft carrier is usually considered by pilots as one of the most difficult exercise, complicated by visibility conditions, carrier dynamics and small landing area [1].

Considering the nonlinear, complexity and fuzziness of Landing Signal Officer, traditional control manner is not fit for "LSO-pilot-aircraft" system [2-4]. To improve control efficiency, a variable universe adaptive landing fuzzy controller should be presented. It establishes an asymmetric one as upward and downward boundaries of Safe Flight Area.

The rest of this paper is structured as follows: next section we first analyse asymmetric variable universe adaptive fuzzy controller. Section 3 designs the Safe Flight Area during approach. Multiple loading conditions simulation results show that corrective strategies is in line with the actual situation of carrier-based aircraft landing in Section 4.

\section{Asymmetric Variable Universe Adaptive Fuzzy Controller}

\subsection{Design Universe-conversion Factors}

Definition 1. Suppose $X I$ is a standard universe, where $X I$ with symmetric unit range $X I=[-1,1]$.

Definition 2. Since $X I$ is a standard universe, function $\gamma: X I \rightarrow X, \quad x i \rightarrow x=\gamma(x i)$ is a universe-conversion factor of the standard universe $X I$, if it satisfies the following axioms: 
(1) Negative weighting: $\forall x i \in X I$, if $x i<0$, then $x=\gamma(x i)=C_{1}(x i)$.

(2) Positive weighting: $\forall x i \in X I$, if $x i \geq 0$, then $x=\gamma(x i)=C_{2}(x i)$.

Where $C_{j}(x i)=C_{j} x i,(j=1,2)$ is converted function which adopted multiple weighted ways, and an ordinary universe $X=\gamma(X I)=\left[-C_{1}, C_{2}\right]$ could be obtained from a standard one with universe-conversion factor.

Definition 3. Suppose an ordinary universe $X=\left[-C_{1}, C_{2}\right]$, we call it is a symmetric universe where $C_{1}=C_{2}$; else an asymmetric one.

If $C_{1} / C_{2}>1, X$ is a negative asymmetric universe;

If $C_{1} / C_{2}<1, X$ is a positive asymmetric universe;

If $C_{1} / C_{2}=1, X$ is a symmetric universe.

Definition 4. Suppose an ordinary universe $X=\left[-C_{1}, C_{2}\right]$, we call $\eta$ is universe ratio item, which is indicated as:

$$
\eta(x)=\left\{\begin{array}{cr}
1, & x \geq 0 \\
C_{1} / C_{2}, & x<0
\end{array}\right.
$$

Then

$$
X=\eta(x)\left[-C_{I}, C_{I}\right]=\left[-\eta(x) C_{I}, \eta(x) C_{I}\right]=\left[-\eta(x) C_{I}, C_{I}\right]
$$

Where $C_{I}$ is standard universe range of $X=\left[-C_{1}, C_{2}\right]$.

\subsection{Design Contraction-expansion Factors}

Generally speaking, a function $\alpha: X \rightarrow[0,1], x \rightarrow \alpha(x)$ is called a universe contraction-expansion factor on ordinary universe $X=\left[-\eta C_{I}, C_{I}\right]$, if it satisfies the following axioms:

(1) Ratio duality: $\forall x \in X, \quad \alpha(\eta(x) x)=\alpha(-\eta(x) x)$;

(2) Zero kept: $\alpha(0)=\delta$;

(3) Monotonicity: $\alpha(x)$ is strictly monotonically increasing on $\left[0, C_{I}\right]$, decreasing on $\left[-\eta C_{I}, 0\right]$;

(4) Compatibility: $\forall x \in X, \quad|x| \leq \alpha(\eta(x) x)\left(\eta C_{I}\right)$;

(5) Normality: $\alpha\left(C_{I}\right)=\alpha\left(-\eta C_{I}\right)=1+\delta$.

Where $\delta$ is positive number with enough small, generally $\delta \leq \min \left[\eta C_{I}, C_{I}\right] / 1000$.

Denote sample steps to be $k=0,1,2, \ldots$.,Contraction-expansion factor of the universe is:

$$
\alpha_{i}\left(x_{i}^{k}\right)=\left\{\begin{array}{cc}
1, & k=0 \\
\left|x_{i}^{k} / \eta_{i}(x) C_{I}\right|^{\tau}+\delta, & k>0
\end{array}\right.
$$


Where $\tau \in(0,1)$. If $X=[-E, E]$ is a symmetric universe, contraction-expansion factor could be denoted by

$$
\alpha_{i}\left(x_{i}^{k}\right)=\left\{\begin{array}{cc}
1, & k=0 \\
\left|x_{i}^{k} / E\right|^{\tau}+\delta, & k>0
\end{array}\right.
$$

And the structure of a variable universe adaptive fuzzy controller is expressed in Fig.1 [5-8].

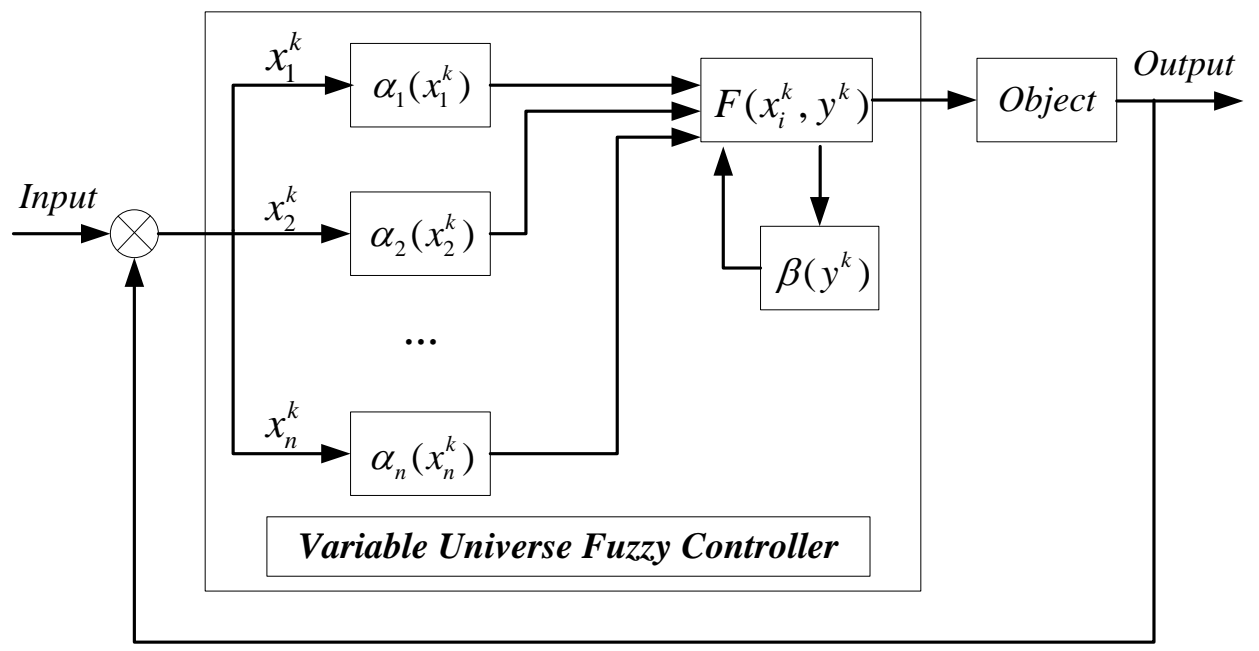

Figure 1. Variable Universe Fuzzy Controller

\subsection{Design Control Algorithm of Asymmetric Variable Universe Fuzzy System}

Let $U_{i}^{0}=\left[-\eta C_{I i}, C_{I i}\right]$ be initial input universe of controller on the moment $k=0$, $V^{0}=\left[-\xi Y_{I}, Y_{I}\right]$ be initial output universe.

Variable universe is the universe of $U_{i}^{k}$ and $V^{k}$ varies with the change of $x_{i}$ and $y$.

$$
\begin{gathered}
U_{i}^{k}=\left[-\eta C_{I i} \alpha_{i}\left(x_{i}^{k}\right), C_{I i} \alpha_{i}\left(x_{i}^{k}\right)\right] \\
V^{k}=\left[-\xi Y_{I} \beta\left(y^{k}\right), Y_{I} \beta\left(y^{k}\right)\right]
\end{gathered}
$$

The linguistic variables are varying together with the varying of the universe, as shown in Fig2. The variable universe adaptive landing fuzzy systems can be written as shown:

$$
y^{k+1}=f\left(\boldsymbol{x}^{k}\right)=\beta\left(y^{k}\right) \frac{\sum_{j=1}^{m} \bar{y}_{j}^{0} \prod_{i=1}^{n} \mu_{A_{i j}^{0}}\left(\frac{x_{i}^{k}}{\alpha_{i}\left(x_{i}^{k}\right)}\right)}{\sum_{j=1}^{m} \prod_{i=1}^{n} \mu_{A_{i j}^{0}}\left(\frac{x_{i}^{k}}{\alpha_{i}\left(x_{i}^{k}\right)}\right)}
$$

With the operation of $\alpha_{i}\left(x_{i}^{k}\right)$ and $\beta\left(y^{k}\right)$, the input variable universe $U_{i}^{k}$ and the output variable universe $V^{k}$ shrink or expand, and the shape of membership functions $\mu_{A_{i j}^{0}}$ becomes narrow or wide, as shown in Fig. 2 [9-18]. 


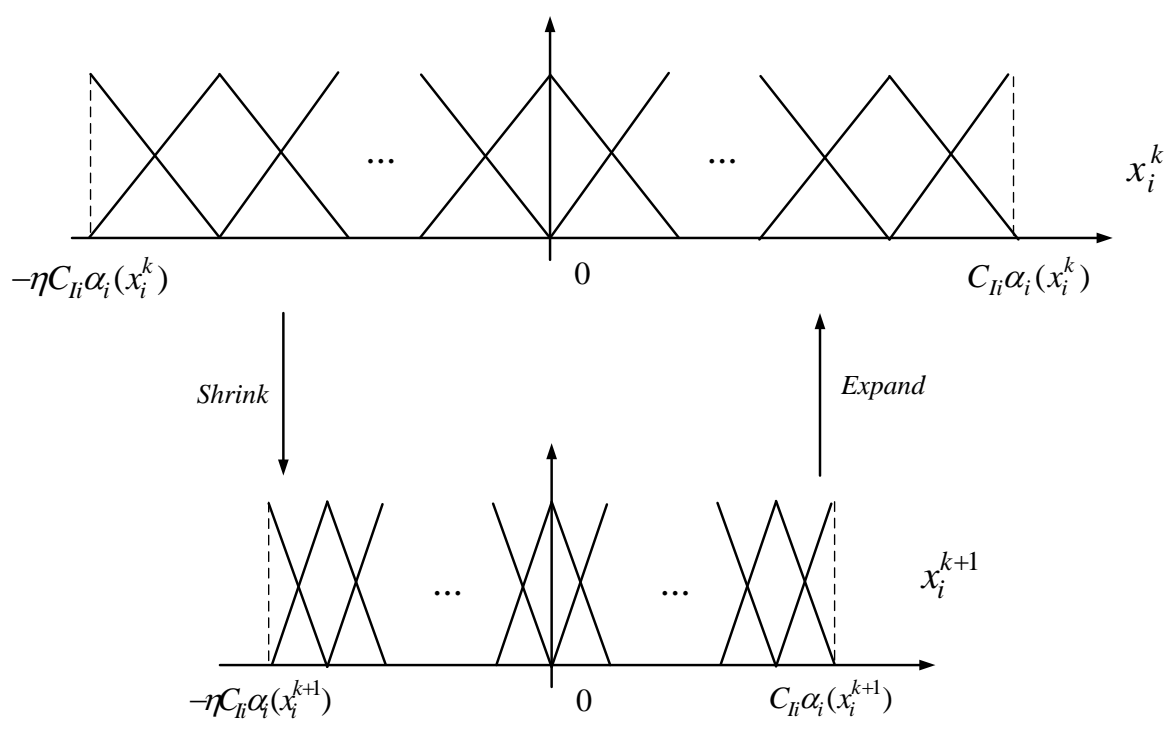

Figure 2. Shrinkage and Expansion of Universe

\section{Safe Flight Area of Carrier-based Aircraft}

Considering the distribution of touchdown points and hook-to-ramp clearance, Safe Flight Area envelope at different reference points will be established. In order to expound the method, IC point should be an example [3-4].

For the simulation, we consider aircraft position from the carrier is equal to 463 meters (IC point), desired velocity and angle of attack are respectively equal to 69.96 meters per second and 8.1 degrees. Preliminary glideslope deviations are varied in the range of \pm 16 meters. 32 groups of the flight trajectories and touchdown points distribution are represented on Fig. 3.

As presented in Fig. 3, According to the distribution of four cables, we directly obtain the longitudinal safe rectangle area on deck of carrier: $W_{\text {Deck }} \in[-18,30] \mathrm{m}$. There is a risk when touchdown point is out of the Safe Rectangle Area, so preliminary deviations should be counted by the range of touchdown points as presented in Fig. 4. The longitudinal Safe Flight Area at IC point is equal to: $z_{p I C} \in[-15.2,11.9] \mathrm{m}$. To ensure the least clearance being 3 meters, the preliminary deviation at IC position is equal to: $z_{p I C}>-15.1 \mathrm{~m}$. Considering the distribution of touchdown and hook-to-ramp clearance, the longitudinal Safe Flight Area at IC point is equal to: $z_{p I C} \in[-15.1,11.9] \mathrm{m}$.
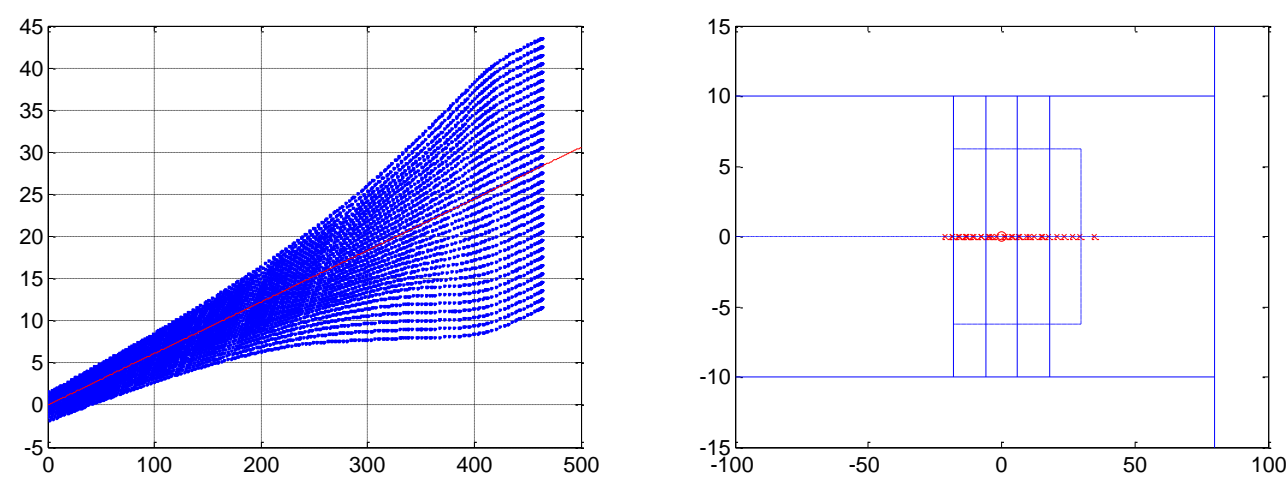

Figure 3. Flight Trajectories on IC Position and Distributions of Touchdown Points 

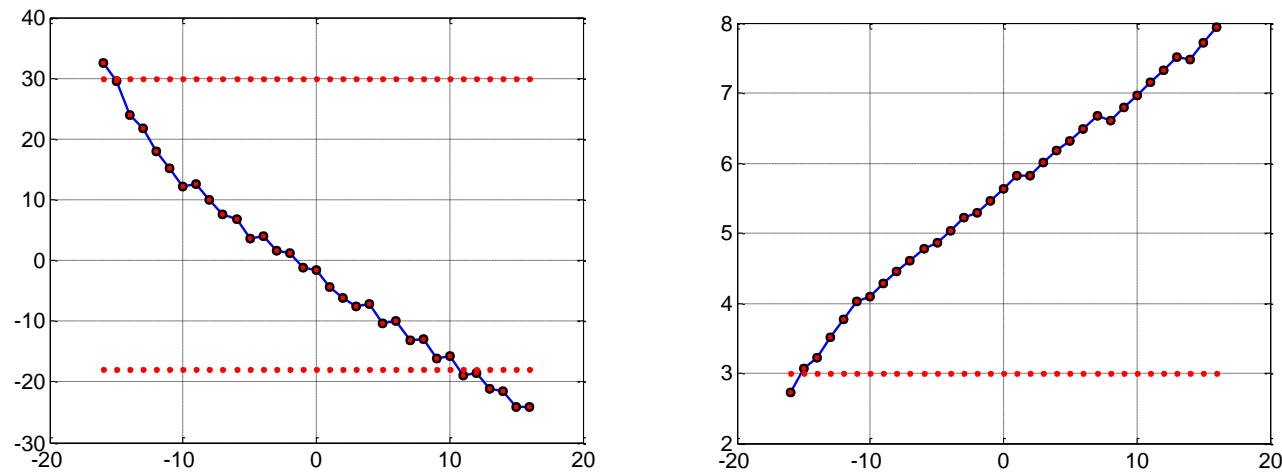

Figure 4. Relationship of Touchdown Points/ Hook-to-ramp Clearances and Deviations

Similar schemes are applied to the other reference points (X, IM, AR) as presented in Table 1.

Table 1. Safe Flight Area on Reference Positions

\begin{tabular}{c|c|c|c|c}
\hline Reference points & Down envelope & Up envelope & Ramp Clearance & SFA boundary \\
\hline $\mathrm{X}(3 / 4 \mathrm{~nm})$ & $-43.5 \mathrm{~m}$ & $39.6 \mathrm{~m}$ & $>-42.8 \mathrm{~m}$ & {$[-42.8,39.6] \mathrm{m}$} \\
\hline $\mathrm{IM}(1 / 2 \mathrm{~nm})$ & $-29.2 \mathrm{~m}$ & $24.4 \mathrm{~m}$ & $>-27.7 \mathrm{~m}$ & {$[-27.7,24.4] \mathrm{m}$} \\
\hline $\mathrm{IC}(1 / 4 \mathrm{~nm})$ & $-15.2 \mathrm{~m}$ & $11.9 \mathrm{~m}$ & $>-15.1 \mathrm{~m}$ & {$[-15.1,11.9] \mathrm{m}$} \\
\hline $\mathrm{AR}(80 \mathrm{~m})$ & $-1.7 \mathrm{~m}$ & $2.6 \mathrm{~m}$ & $>-1.9 \mathrm{~m}$ & {$[-1.7,2.6] \mathrm{m}$} \\
\hline
\end{tabular}

\section{Model Simulation}

To improve the tracking precision of aircraft's trajectory, it designs Single-Input and Double-Output asymmetric variable universe adaptive fuzzy landing control system as shown in Fig.5.

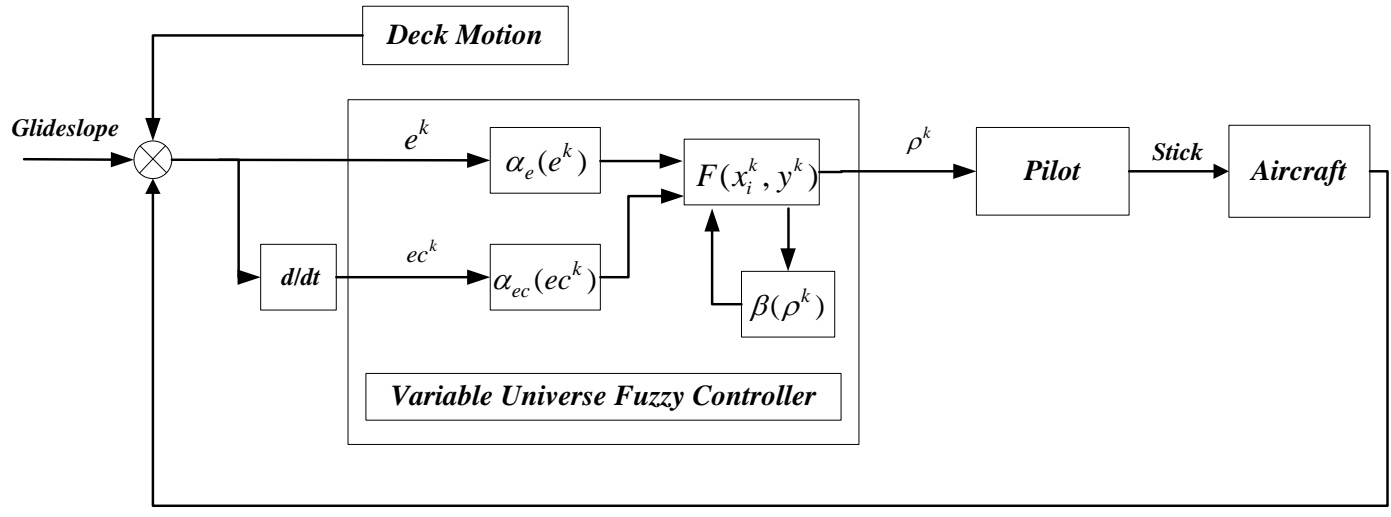

\section{Figure 5. Variable Universe Fuzzy Trajectory Controller of Carrier-based Aircraft}

The control process could be realized for some steps as follow:

Step 1. Initial universe of fuzzy control system: As safe flight area for carrier-based aircraft, $S_{F S A}=[-27.7,24.4] \mathrm{m}$ at reference point IM. It makes that universe ratio item $\eta=27.7 / 24.4=1.135$, and the universe of $e^{0}$ is $X_{e}^{0}=[-27.7,24.4]$, the 
universe of $e c^{0}$ is $X_{e c}^{0}=[-\eta \times 5,5]=[-5.675,5]$, the universe of $\rho^{0}$ is $Y^{0}=[-3,3]$,

Step 2. Fuzzy division of control system: $\bar{x}_{1 j}^{0}(j=1,2, \ldots, 6)$ where fuzzy peak points on $e^{0}$ are divided at $-27.7,-17.28,-6.86,3.56,13.98,24.4 ; \bar{x}_{2 j}^{0}(j=1,2, \ldots, 6)$ where fuzzy peak points on $e c^{0}$ are divided at $-5.675,-3.54,-1.405,0.73,2.865$ , 5 .

Step 3. Membership functions: Using triangle membership functions, we define $\mu_{A_{1 j}^{0}}(j=1,2, \ldots, 6)$ for $X_{e}^{0}$ as follows:

$$
\begin{aligned}
& \mu_{A_{11}^{0}}\left(e^{0}\right)=\left\{\begin{array}{cc}
\left(e^{0}+17.28\right) /(-27.7+17.28) & -27.7 \leq e^{0}<-17.28 \\
0 & \text { else }
\end{array}\right. \\
& \mu_{A_{12}^{0}}\left(e^{0}\right)=\left\{\begin{array}{cc}
\left(e^{0}+27.7\right) /(27.7-17.28) & -27.7 \leq e^{0}<-17.28 \\
\left(e^{0}+6.86\right) /(-17.28+6.86) & -17.28 \leq e^{0}<-6.86 \\
0 & \text { else }
\end{array}\right. \\
& \ldots \ldots
\end{aligned}
$$

$\mu_{A_{2 j}^{0}}(j=1,2, \ldots, 6)$ for $X_{e c}^{0}$ as follows:

$$
\begin{aligned}
& \mu_{A_{21}^{0}}\left(e c^{0}\right)=\left\{\begin{array}{cc}
\left(e c^{0}+3.54\right) /(-5.675+3.54) & -5.675 \leq e c^{0}<-3.54 \\
0 & e l s e
\end{array}\right. \\
& \mu_{A_{12}^{0}}\left(e c^{0}\right)=\left\{\begin{array}{cc}
\left(e c^{0}+5.675\right) /(5.675-3.54) & -5.675 \leq e c^{0}<-3.54 \\
\left(e c^{0}+1.405\right) /(-3.54+1.405) & -3.54 \leq e c^{0}<-1.405 \\
0 & e l s e
\end{array}\right. \\
& \mu_{A_{26}^{0}}\left(e c^{0}\right)=\left\{\begin{array}{cc}
\left(e c^{0}-2.865\right) /(5-2.865) & 2.865<e c^{0} \leq 5 \\
0 & e l s e
\end{array}\right.
\end{aligned}
$$

Step 4. Contraction-expansion factors:

$$
\begin{aligned}
& \alpha_{e}\left(e^{k}\right)=\left\{\begin{array}{cc}
1 & k=0 \\
\left(\left|e^{k}\right| / 27.7\right)^{0.95}+0.01 & k=1,2,3, \ldots
\end{array}\right. \\
& \alpha_{e c}\left(e c^{k}\right)=\left\{\begin{array}{cc}
1 & k=0 \\
\left(\left|e c^{k}\right| / 5.675\right)^{0.95}+0.01 & k=1,2,3, \ldots
\end{array}\right.
\end{aligned}
$$




$$
\beta\left(\rho^{k}\right)=\left\{\begin{array}{cc}
1 & k=0 \\
\left(\left|\rho^{k}\right| / 3\right)^{0.95}+0.01 & k=1,2,3, \ldots
\end{array}\right.
$$

Step 5. Fuzzy Controller is defined as follow:

$$
\rho^{k+1}=\beta\left(\rho^{k}\right) \frac{\sum_{j=1}^{36} \bar{\rho}_{j}^{0} \mu_{A_{1 j}^{0}}\left(\frac{e^{k}}{\alpha_{e}\left(e^{k}\right)}\right) \mu_{A_{2 j}^{0}}\left(\frac{e c^{k}}{\alpha_{e c}\left(e c^{k}\right)}\right)}{\sum_{j=1}^{36} \mu_{A_{1 j}^{0}}\left(\frac{e^{k}}{\alpha_{e}\left(e^{k}\right)}\right) \mu_{A_{2 j}^{0}}\left(\frac{e c^{k}}{\alpha_{e c}\left(e c^{k}\right)}\right)}
$$

Fig.6-10 are the response curves of flight path, height deviation, longitudinal sick, gliding angle and velocity for different control patterns.

From Fig.6-10, the approximation error of system is $\varepsilon=0.2 \mathrm{~m}$ at the moment $3.8 \mathrm{~s}$ under the asymmetric variable universe adaptive fuzzy landing control system, and it achieves the same error at the moment $12.3 \mathrm{~s}$ under the traditional landing control system. It has the superiority complex on approximation error for the asymmetric variable universe adaptive fuzzy landing control system we designed.

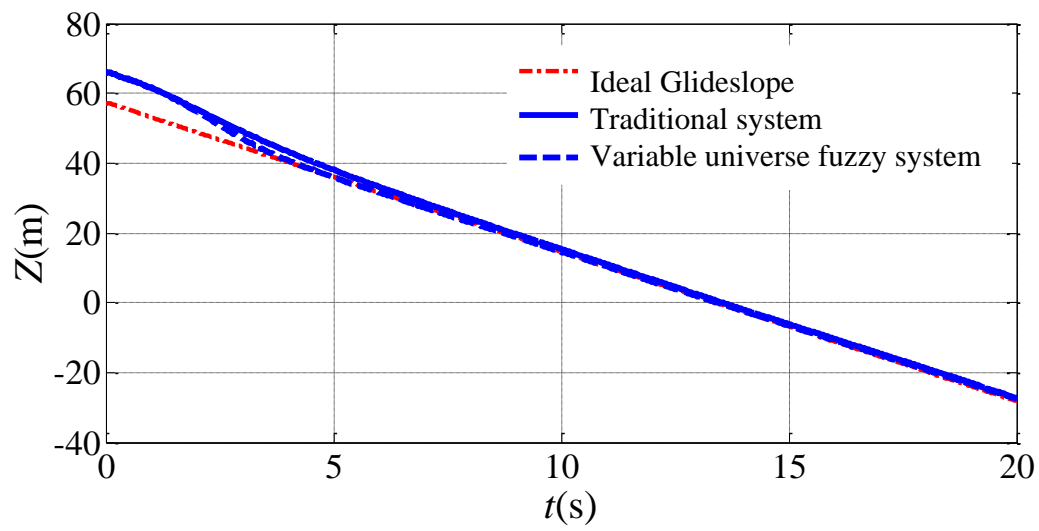

Figure 6. Response Curve of Flight Path

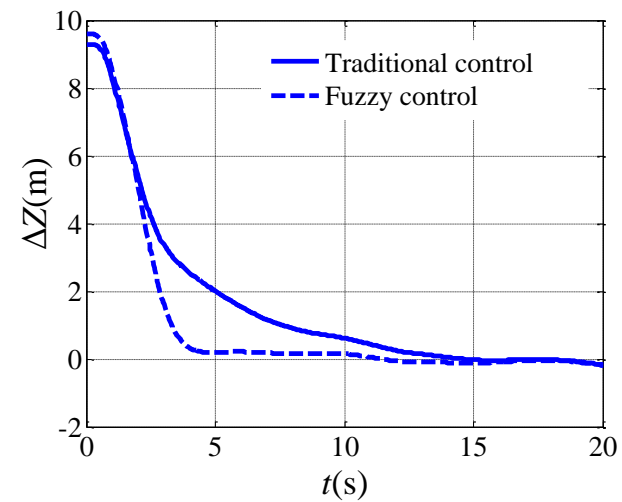

Figure 7. Response Curve of Deviation

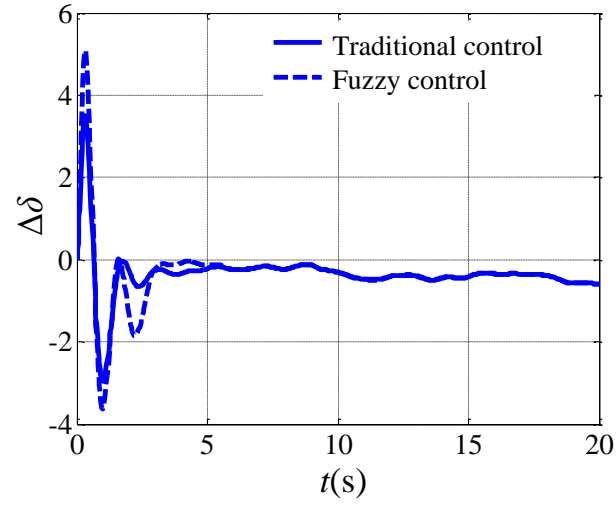

Figure 8. Response Curve of Stick 


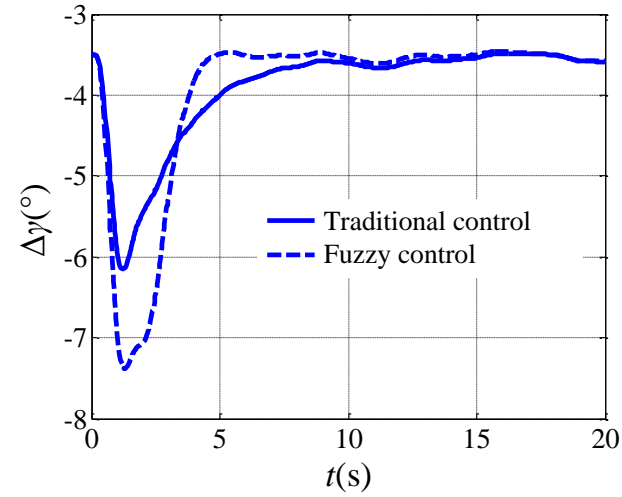

Figure 9. Response Curve of Gliding Angle

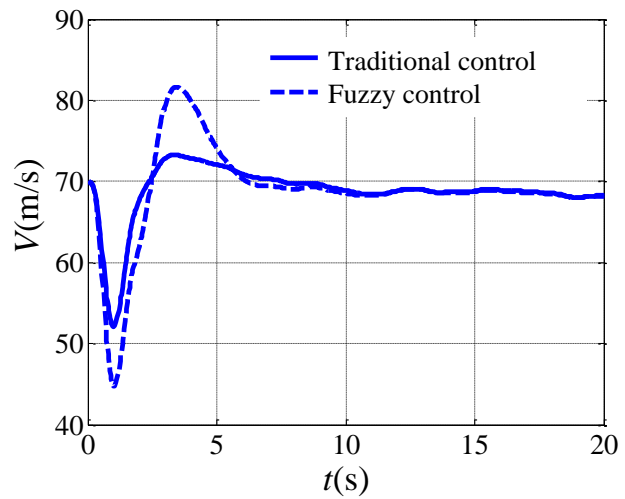

Figure 10. Response Curve of Velocity

\section{Conclusion}

This paper has presented an improved control manner for landing approach to perform manual landing on aircraft carrier. This asymmetric variable universe adaptive landing fuzzy controller with Safe Flight Area is established with universeconversion factors and contraction-expansion factor. The simulation results show that compared with the traditional control manner, the fuzzy method with the SFA envelope has better evaluation result for pilots, which means more accuracy and dynamic.

\section{Acknowledgement}

The author would like to thank the anonymous referees for their valuable suggestions. This work was supported by the Natural Science Foundation of Heilongjiang Province of China (Grant Nos. F201349), Natural Science Foundation of Heilongjiang Province of China (Grant Nos. F201423), Natural Science Foundation of Heilongjiang Province of China (Grant Nos. F201426), Science and Technology Research Foundation of Heilongjiang Education Department (Grant Nos. 12531162) and the Natural Science Foundation of Heilongjiang Province of China (Grant Nos. F201210).

\section{References}

[1] R. Richards, “Artificial Intelligence Techniques for Pilot Approach Decision Aid Logic System", Stottler Henke Associates, Inc., (2000).

[2] T. Rudowsky, S. Cook and M. Hynes, "Review of the carrier approach criteria for carrier-based aircraft", Techinical report, (2002).

[3] R. B. Johnstone, "Development of the wave-off decision device and its relationship to the carrier approach problem", AIAA-68-846, American Inst of Aeronautics and Astronautics, Guidance, Control, and Flight Dynamics Conference, (1968); Pasadena, Calif..

[4] H. X. Li, Z. H. Miao and J. Y.Wang, "Variable Universe Adaptive Fuzzy Control on the Quadruple Inverted Pendulum", Science in China (Series E), vol. 45, no. 2, (2002), pp. 213-224.

[5] L. X. Wang, "Fuzzy systems are universal approximator", Proceedings of IEEE International Conference on Fuzzy Systems, (1992); San Diego, USA.

[6] W. W. Shan, Y. Ma and R. W. Newcob, "Analog Circuit Implementation of a Variable Universe Adaptive Fuzzy Logic Controller", IEEE Transactions on Circuits and Systems-II: Express Briefs, vol. 55, no. 10, (2008), pp. 976-980.

[7] L. F. Li, X. Y. Liu and W. F. Chen, "A Variable Universe Fuzzy Control Algorithm Based on Fuzzy Neural Network", Proeeedings of the $7^{\text {th }}$ World Congress on Intelligent Control and Automation, (2008); Chongqing.

[8] J. Wang and W. W. Zhang, "Chaos Control via Variable Universe Fuzzy Theory in Auto Gauge Control System", International Conference on Computer Application and System Modeling, (2010); Taiyuan.

[9] H. Ying, "General Takagi-Sugeno Fuzzy Systems with Simplified Linear Rule Consequent as Universal Controllers", Models and Filters, IEEE Transation on Fuzzy Systems, no. 108, (1998), pp. 91-107. 
[10] K. Zeng, N. Y. Zhang and W. L. Xu, "Comparative Study on Sufficient Conditions for Takagi-Sugeno Fuzzy Systems as Universal Approximators”, IEEE Transactions on Fuzzy Systems, vol. 8, no. 6, (2000), pp. 773-780.

[11] R. Hassine, F Karray and A. M. Alimi, "Approximation Properties of Piecewise Parabolic Functions Fuzzy Logic Systems”, Fuzzy Sets and Systems, vol. 35, no. 157, (2006), pp. 501-515.

[12] P. Y. Liu and H. X. Li, "Hierarchical TS Fuzzy System and Its Universal Approximation", Information Sciences, vol. 34, no. 169, (1974), pp. 279-303.

[13] R. M. Robertson, D. W. Maxwell and C. E. Williams, "The Landing Signal Officer: Auditory Aspects", no. 12 , (2005), pp. 4-21.

[14] M. G. Joo and J. S. Lee, "Universal Approximation by Hierarchical Fuzzy System with Constraints on the Fuzzy Rule", Fuzzy Sets and Systems, no. 130, (2002), pp. 175-188.

[15] D. C. Li, Z. K. Shi and Y. M. Li, "Sufficient and Necessary Conditions for Boolean Fuzzy Systems as Universal Approximators", Information Science, vol. 178, no. 2, (2008), pp. 414-424.

[16] Y. M. Li, Z. K. Shi and Z. H. Li, "Approximation Theory of Fuzzy Systems Based upon Genuine Manyvalued Implications-MIMO Cases”, Fuzzy Sets and Systems, vol. 130, no. 2, (2002), pp. 159-174.

[17] C. Guo, J. X. Zhao and Z. Q. Chen, "H-infinity Variable Universe Fuzzy Control for Hyseretic Systems", Journal of University of Science and Technology of China, vol. 37, no. 9, (2007), pp. 1130-1136.

[18] J. Wang, G. D. Qiao and B. Deng, "Observer-based Robust Adaptive Variable Universe Fuzzy Control for Chaotic System”, Chaos, Solutions \& Fractals, vol. 23, (2005), pp. 1013-1032.

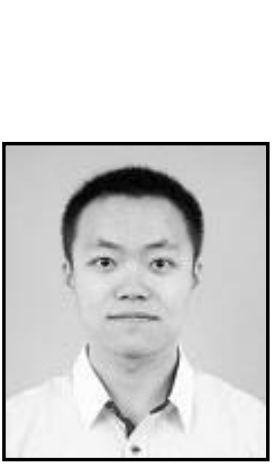

\section{Authors}

Hui Li, he received a D.E. degree in Control Theory and Control Engineering from Harbin Engineering University, Harbin, China, 2013. He is the member of council of the Operations Research Society of China. His recent research interests are in intelligent control, Multi-attribute decision making, fuzzy decision making.

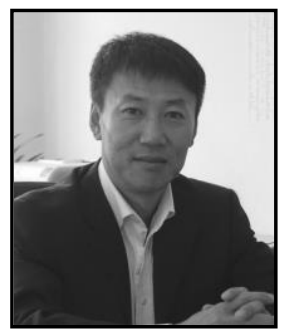

Xiao-dong Su, he is an professor of Harbin University of Commerce and he is the member of council of the China Software Industry Association, vice president of Electronics Experiment Teaching Research Council of Universities in Heilongjiang, member of Heilongjiang Computer Society Education and Training Professional Committee. His recent research interests are in information detection and estimation, management information systems.

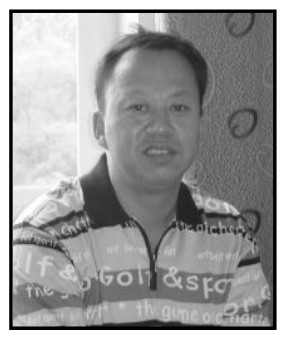

Hai-tao Jiang, he is an associate professor of Harbin University of Commerce. He received a M.E. degree in Software Engineering from Beijing University of Posts and Telecommunications, Beijing, China, 2010. His recent research interests are in intelligent control, digital signal processing and embedded technology. 
International Journal of Smart Home

Vol. 9, No. 5 (2015) 